\title{
Analysis of multiple solutions in seismic facies inversion for complex geology
}

\author{
Erick Costa e Silva Talarico, Petrobras S.A.
}

Dario Grana, Wyoming University

Leandro Passos de Figueiredo, LTrace and UFSC

Sinesio Pesco, Pontifical Catholic University - Rio de Janeiro

Copyright 2019, SBGf - Sociedade Brasileira de Geofísica

This paper was prepared for presentation during the $16^{\text {th }}$ International Congress of the Brazilian Geophysical Society held in Rio de Janeiro, Brazil, 19-22 August 2019.

Contents of this paper were reviewed by the Technical Committee of the $16^{\text {th }}$ International Congress of the Brazilian Geophysical Society and do not necessarily International Congress of the Brazilian Geophysical Society and do not necessarily
represent any position of the SBGf, its officers or members. Electronic reproduction or represent any position of the SBGf, its officers or members. Electronic reproduction or
storage of any part of this paper for commercial purposes without the written consent of the Brazilian Geophysical Society is prohibited.

\begin{abstract}
Facies inversion is a class of algorithms, where the inversion is written in terms of facies, in addition to the usual acoustic (or elastic) parameters. Alike the other approaches, facies inversion needs some parametrization, which depends on physical and geological reasoning as well as specialist experience. This work discusses the problem of inferring the inversion parameters from the seismic data itself. The parametrization of the geological related parameter, namely, transition probability matrix, is inferred given the seismic data, using Bayesian framework. While it is usually assumed that a single parameter value can optimally fit the measurements, in the present paper, this hypothesis is questioned. In both synthetic and real seismic datasets it is shown that the symmetries of the convolutional model allow more than one transition matrix to be a local optimum to the inference problem, which enables a higher diversity of possible solutions to the inversion problem than usual approaches.
\end{abstract}

\section{Introduction}

The facies classification from seismic in hydrocarbon reservoirs is a challenging problem since seismic data is an indirect measurement, with limited resolution and usually low signal to noise ratio. Some authors have approached the problem from a Bayesian inverse theory point of view [1-2], others with geostatistical tools [3-9] and yet other by means of machine learning [10-11].

The solution of the inversion problem is not unique because different facies sequences may generate indistinguishable seismic response. Thus, uncertainty quantification in facies inversion is as important as the prediction of the most likely facies sequence.

Bayesian methods have been widely applied to several geophysical inverse problems $[2,12,13,14]$, and aim to predict a probability distribution of the variable of interest, given a set of hyperparameters. In this approach, the variable of interest is described by a prior probability distribution before the measurement is made. Through the comparison of the modelled signal and the measurement (in the likelihood distribution), one can assess the posterior probability distribution, i.e. the variable's probability after the measurement is taken into account.

The facies distribution in the subsurface is assumed spatially correlated due to the geological continuity of the deposition and expected stratigraphic stacking. The seismic likelihood also adds spatial correlation due to resolution limitations. Approaches with spatial dependency between properties were studied in $[1,15,16,17,18,19,20]$, where the prior distribution of the possible facies sequences is described using a first order Markov chain, parametrized by a transition probability matrix of the facies, usually estimated from nearby wells.

Some authors [21-22] estimate the transition matrix from the seismic data alone, by maximizing the seismic likelihood as a function of the transition matrix. They assume that the seismic measurements themselves are enough to estimate the true transition matrix from all other possibilities. This might not be the case in some situations, as shown in the present paper: multiple local maxima to the likelihood function might exist. The presented methodology is important in complex geologies, such as carbonates, since it can sample more possibilities of facies sequences than the approaches from the previous papers.

The Method section describes the inversion algorithm and the Bayesian inference. The Examples section presents both synthetic and real seismic dataset to validate the methodology.

\section{Method}

The facies inversion technique used in this paper was developed in [22-23], i.e. the inversion problem is written as a convolved hidden Markov model.

The facies sequence is denoted by $\pi=\left(\pi_{1}, \pi_{2}, \ldots, \pi_{N}\right)$, and facies $\pi_{n}$ at position $n$ can be of any the $\mathrm{K}$ types defined by the geoscientists $\pi_{n} \in \Omega=\{1, \ldots K\}$. The seismic trace is denoted by $\boldsymbol{s}$, and is zero-offset.

Acoustic impedance, the link between facies and seismic, is denoted by $I_{P}$. By assuming the weak contrast approximation $s_{n}=\log I_{P n}-\log I_{P, n-1}$ introduced in [24], the logarithm of the acoustic impedance is used instead $\boldsymbol{m}=\left(\log I_{P 1}, \log I_{P 2}, \ldots, \log I_{P N}\right)$.

The likelihood of the seismic trace $s$ given facies sequence $\pi$ is defined as $p(s \mid \pi)=\mathcal{N}\left(s \mid G \mu_{\pi}, \mathrm{G} \Sigma_{\pi} \mathrm{G}^{\mathrm{T}}+\Sigma_{e}\right)$, where $G$ is the convolutional forward model, $\mu_{\pi}$ is the mean logimpedance sequence, $\Sigma_{\pi}$ is the corresponding covariance matrix, and $\Sigma_{e}$ is the seismic noise covariance matrix.

The facies prior distribution is given by a first order Markov Chain. It is defined by the equation $p\left(\pi_{n}=j \mid \pi_{n-1}=i\right)=$ $T_{i j}$, where $T$ is the transition matrix. This parameter 
controls the stratigraphic patterns that are present in facies sequences.

From Bayes rule, the posterior probability $p(\pi \mid s)$ of the facies sequences given seismic trace is proportional to the product of the prior distribution and the likelihood. From this equation, the inversion problem can be translated as trying to find facies sequences that model the measured seismic data and at the same time respect the expected stratigraphic style. The posterior distribution $p(\pi \mid s)$ is complex with long range dependency between facies samples at different positions, and there is no analytical solution. Some authors $[16,18,21,22]$ solve the facies inversion problem by approximating this posterior distribution as a product of factors $p(\pi \mid s) \approx$ $\prod_{n} q\left(\pi_{n-k}, \ldots, \pi_{n+k}\right)$, each one containing a facies template of only $2 k$ samples. This new probability distribution can be interpreted as a hidden Markov model on the facies template variable $z_{n}=\left(\pi_{n-k}, \ldots, \pi_{n+k}\right)$, thus it can use the recursive solution developed for this class of problem [25]. The approximation is better for bigger $k$, but the computational cost also increases because the number of facies templates of size $2 k$ increases exponentially as $K^{2 k}$.

In this paper, the above inversion approximation is used, but it is encouraged that other algorithms are also possible. Now, the transition matrix inference problem can be explained.

The transition matrix likelihood can be defined as $p(s \mid T)=$ $\sum_{\pi} p(s \mid \pi) p(\pi \mid T)$. This equation can be interpreted as the correlation coefficient between $p(s \mid \pi)$ and $p(\pi \mid T)$, i.e. the transition matrix with big probability on facies sequences that best model the seismic sequence has highest likelihood. Given no prior information about the transition matrix, the transition matrix can be inferred from the seismic data by maximizing the likelihood function. While some authors assume that the likelihood function has a single optimum, this paper will explore the possibility of there being more than one local maxima, which should be considered a valid solution.

Finally, Chapman-Kolmogorov equation can be used to invert the seismic data to facies sequences considering the uncertainty in transition matrix: $p(\pi \mid s)=\sum_{T} p(\pi \mid s, T) p(T \mid s)$

\section{Examples}

A first order Markov Chain with two facies types and a uniform transition matrix is sampled. The corresponding log-impedance distributions are shown in Figure 1 as facies 1 and 2 . The synthetic seismogram is illustrated in Figure 3 . The wavelet is a Dirac delta, so in this example the methodology is validated with an inversion from reflectivity to facies.

Just two facies are observable at the well location, but from the symmetry between the mean log-impedances, in Figure 1, the seismogram can be either explained by a sequence of facies 2 and 3 , or facies 1 and 2 . This example illustrates the situation where some facies were not drilled in a well but it are known to exist in the area.

A random search in transition matrix space revealed two local maxima with approximately equal likelihoods, as shown in Figure 2. As expected, one local maximum corresponds to the correct facies sequence, and the other

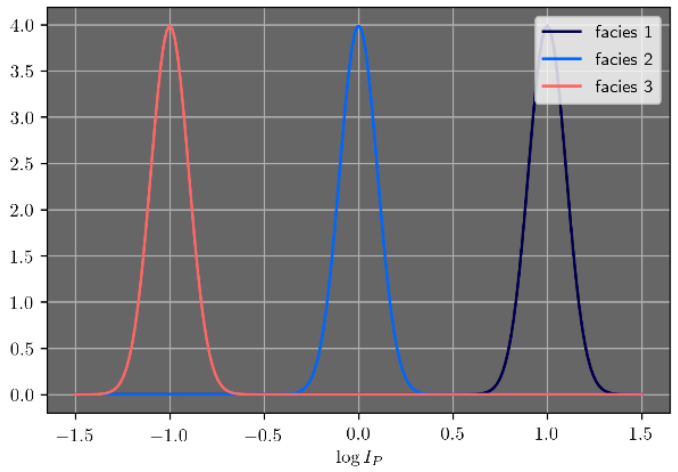

Figure 1: Synthetic log-impedance prior distribution color coded by facies.

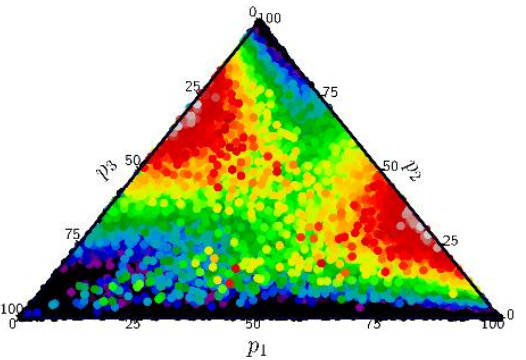

Figure 2: Likelihood function (logarithmic scale) represented on a ternary plot. Each point in the plot represents a transition matrix, projected on the space of stationary probabilities for simpler visualization.

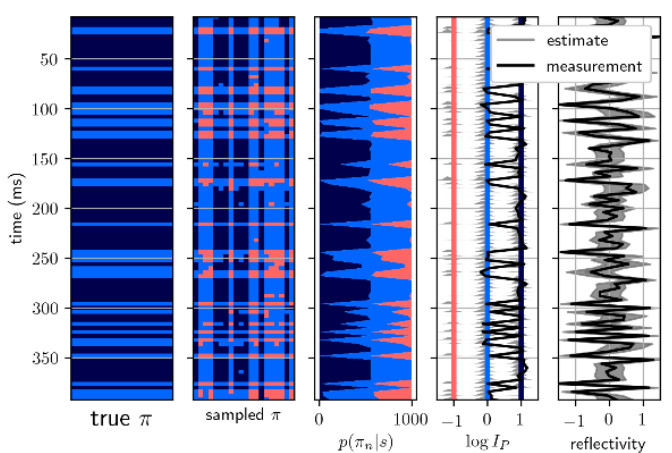

Figure 3: In the first track, the true facies profile. In the second track, the sampled facies sequences (20 sequences side by side). In the third track, the posterior facies probabilities. In th forth track, the true logimpedance log in black, and the posterior log-impedance histograms in gray. In the last track the measured reflectivity in black and the modelled $80 \%$ confidence region in gray.

corresponds to a sequence with only facies 2 and 3 . Figure 3 shows the result of Chapman-Kolmogorov equation to this example. Should just one transition matrix be considered, much less variability would exist in the facies realizations, since just sequences of facies 1 and 2 or 2 and 3 would be sampled, depending on which local maxima is used for the inversion.

As a second example, a real dataset, from a carbonate play is presented. There are three possible facies, closed 
carbonate, porous carbonate, and super-porous carbonate. Seismic section in Figure 4 shows how facies varies laterally in a carbonate play. In well $\mathrm{W} 1$, the porous rock is the background rock, while in well W2, closed carbonates are the background rock. The challenge in carbonate plays is to detect super porous carbonates, at a distant position, where the background is unknown.

The seismic trace at well W1 position Figure 4 is used in the example. A random search is done in the transition matrix space, revealing 5 local maxima. The facies probabilities for each transition matrix are shown in Figure 5. In this case, the different local maxima have very different values of likelihood, possibly due to the facies inversion approximation, since the approximation is worst when the wavelet is regular sized.

\section{Conclusions}

In this work, a new uncertainty quantification workflow for facies inversion is presented. It is shown that the seismic likelihood given transition matrix might have multiple local maxima. Each local maximum, when used to parametrize the inversion, generates very different facies realizations. It is also shown how to sample facies realizations when the transition matrix is unknown. The proposed workflow presents a robust uncertainty analysis, and its applicability to risk analysis in complex geology, such as carbonates, is immediate because the fast lateral variation of facies in such plays increases the uncertainty in the facies proportions.

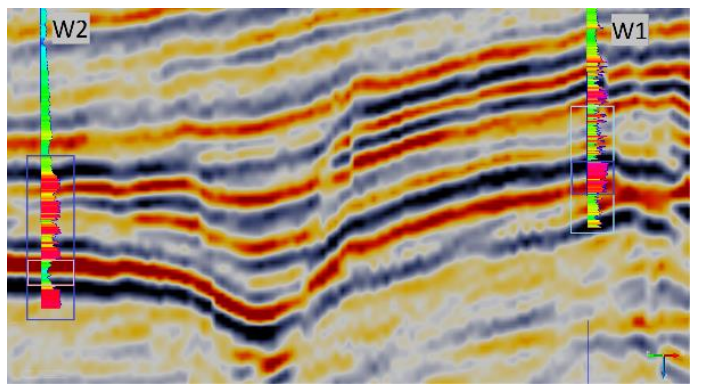

Figure 4: Seismic section illustrating how fast geology change laterally in carbonate plays. Well $W 1$ has a predominance of porous facies, while well W2 has a predominance of closed facies. The impedance log is plotted for each well.

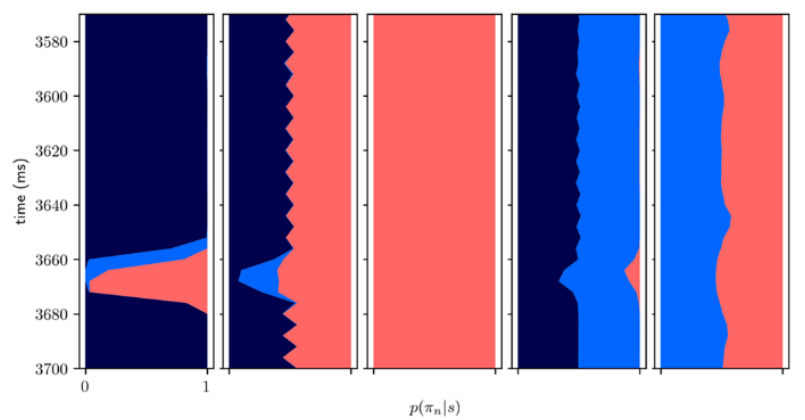

Figure 5: Facies posterior probabilities for each local maximum in likelihood space. Each transition matrix produces a very different inversion result.

\section{Acknowledgments}

The authors would like to acknowledge Petrobras, University of Wyoming and Pontifical Catholic University of Rio de Janeiro for supporting the present work. Acknowledgement to Petrobras for allowing to publish its results. And acknowledgement to Pontifical Catholic University for the financial support.

\section{References}

[1] D. Grana, "Bayesian Inversion Methods for Seismic Reservoir Characterization and Time-Lapse Studies," 2013.

[2] A. Tarantola, Inverse Problem Theory and mMethods for Parameter Estimation, SIAM, 2005.

[3] O. Dubrule, Geostatistics for Seismic Data Integration in Earth Models, SEG Distinguished Instructor Short Course, 2003.

[4] J. Eidsvik, P. Avseth, H. Omre, T. Mukerji and G. Mavko, "Stochastic reservoir characterization using prestack seismic data," Geophysics, vol. 69, pp. 978993, 2004.

[5] P. M. Doyen, Seismic Reservoir Characterization An Earth Modelling Perpective, Education Tour Series - EAGE, 2007.

[6] E. F. Gonzalez, T. Mukerji and G. Mavko, "Seismic inversion combining rock physics and multiple-point geostatistics," Geophysics, vol. 73, pp. R11-R21, 2008.

[7] L. Azevedo, R. Nunes, P. Correia, A. Soares, G. S. Neto and L. Guerreiro, "Stochastic direct facies seismic AVO inversion," Proceedings of 83rd SEG Annual Meeting, 2013.

[8] P. A. Connolly and M. J. Hughes, "Stochastic inversion by matching to large numbers of pseudowells," Geophysics, vol. 81, pp. M7-M22, 2016.

[9] L. Azevedo and A. Soares, Geostatistical methods for reservoir geophysics, Springer, 2017.

[10] B. West, S. May, J. EastWood and C. Rossen, "Interactive seismic facies classification using textural attributes and neural networks," The Leading Edge, vol. 21, pp. 1042-1049, 2002.

[11] B. B. Bougher and F. J. Herrmann, "AVA classification as an unsupervised machine-learning problem," Proceedings of 86th SEG Annual Meeting, 2016.

[12] T. J. Ulrych, M. D. Sacchi and A. Woodbury, "A Bayes tour of inversion: A tutorial," Geophysics, vol. 66, pp. 55-69, 2001.

[13] J. Scales and L. Tenorio, "Prior information and uncertainty in inverse problems," Geophysics, vol. 66, pp. 389-397, 2001.

[14] A. Buland and H. Omre, "Bayesian linearized AVO inversion," Geophysics, vol. 68, pp. 185-198, 2003.

[15] A. L. Larsen, M. Ulvmoen, H. Omre and A. Buland, "Bayesian lithology fluid prediction and simulation on the basis of a Markov-chain prior model," Geophysics, vol. 71, pp. R69--R78, 2006.

[16] M. Ulvmoen and H. Omre, "Improved resolution in Bayesian lithology/fluid inversion from prestack 
seismic data and well observations: Part 1 -Methodology," Geophysics, vol. 75, pp. R21--R35, 2010.

[17] M. Ulvmoen, H. Omre and A. Buland, "Improved resolution in Bayesian lithology/fluid inversion from prestack seismic data and well observations: Part 2 Real case study," Geophysics, vol. 75, pp. B73--B82, 2010.

[18] K. Rimstad and H. Omre, "Approximate Posterior Distributions for Convolutional Two-Level Hidden Markov Models," Computational Statistics and Data Analysis, vol. 53, pp. 187-200, 2013.

[19] D. Grana, T. Fjeldstad and H. Omre, "Bayesian Gaussian Mixture Linear Inversion for Geophysical Inverse Problems," Mathematical Geosciences, vol. 49, pp. 493-515, 2017.

[20] T. Fjeldstad and D. Grana, "Joint probabilistic petrophysics-seismic inversion based on Gaussian mixture and Markov chain prior models," Geophysics, vol. 83, pp. 1-46, 2017.

[21] D. Lindberg and H. Omre, "Bind Categorical Deconvolution in Two-Level Hidden Markov Models," IEEE Transactions on Geoscience and Remote Sensing, vol. 52, pp. 7435-7447, 2014.

[22] T. Fjeldstad, "Bayesian Inversion and Inference of Categorical Markov Models with Likelihood Functions Including Dependence and Convolution," 2015.

[23] E. Talarico, D. Grana, L. P. d. Figueiredo and S. Pesco, "Uncertainty quantification in seismic facies inversion," Mathematical Geoscience, 2019, in preparation.

[24] R. Bortfeld, "Approximations to the reflection and transmission coefficients of plane longitudinal and transverse waves," Geophysical Prospecting, vol. 9, pp. 485-502, 1961.

[25] C. M. Bishop, Pattern Recognition and Machine Learning, Springer, 2006.

[26] E. Talarico, "Seismic to Facies Inversion using Convolved Hidden Markov Model," 2018. 